\title{
Stakeholders knowledge, attitude, and practices (KAP) towards aflatoxins contamination in peanut-based products
}

\begin{abstract}
The aims of this study were to identify the differences in terms of knowledge, attitude and practices (KAP) of aflatoxins contamination amongst stakeholders of peanut-based products and to determine factors that mostly influence stakeholders' hygienic practices in peanutbased products. Using stratified random sampling responses were obtained from a total of 109 stakeholders of peanut-based products companies consisting of importers, small and large-scale manufacturers, and retailers located in Peninsular Malaysia. Statistical analyses including descriptive analysis, mean ranking analysis, one-way ANOVA and logistic regression analysis were used to analyze the data. The results revealed that the stakeholders have adequate knowledge, favorable attitude, and high hygiene practices towards aflatoxins contamination in peanut-based products with mean scores of 2.54, 4.27, and 2.61, respectively. Based on the one-way ANOVA results, there was a significant difference $(\mathrm{p}<$ 0.05) in knowledge and practices among the four different stakeholder groups. However, the result for attitude level towards minimizing aflatoxins contamination was found to be not significantly difference amongst stakeholder groups. Results of the logistic regression analysis showed that four significant variables namely hygiene and training programs, storage, knowledge about aflatoxins, and quality assurance certification were the most influential factors influencing hygiene practices towards minimizing aflatoxins contamination in peanut-based products. The results strongly emphasized the need for continuous hygiene improvement and training programs by the stakeholders of peanut-based products. Relevant strategies such as promotion and motivational models on health education and food safety campaigns will increase awareness and knowledge on food contaminants.
\end{abstract}

Keyword: Knowledge; Attitude; Hygiene practices; Aflatoxins; Peanut; Stakeholders 